Polymer Journal, Vol. 39, No. 11, pp. 1167-1171 (2007)

(C)2007 The Society of Polymer Science, Japan

\title{
A Group Contribution-Based Model to Predict Organic Solvent Diffusivities in Amorphous Rubbery Polymers
}

\author{
Hong-ling Lv, ${ }^{\dagger}$ Bao-guo WANG, and Ji-chu YANG \\ Department of Chemical Engineering, Tsinghua University, Beijing 100084, the People's Republic of China
}

(Received April 15, 2007; Accepted August 10, 2007; Published September 26, 2007)

\begin{abstract}
A modified free-volume model was developed to predict solvent diffusion coefficients in amorphous rubbery polymers, with the introduction of the Sanchez-Lacombe equation-of-state (EOS) into the free-volume theory. The characteristic parameters of the EOS are the only introduced parameters, and they can be determined with the knowledge of polymer structural units. Since all of the parameters with respect to the polymer can be determined by the group contribution method, this model provides feasibility to correlate the solvent diffusivities with polymer structures, without the knowledge of any diffusion or viscoelastic data. The calculations of infinite dilution diffusion coefficients and solvent self-diffusion coefficients for seven kinds of organic solvents in seven kinds of polymers were generally consistent with the published experimental results. [doi:10.1295/polymj.PJ2007011]

KEY WORDS Diffusion Coefficient / Free Volume / Group Contribution / Polymer / Organic

Solvent / Equation-of-State (EOS) /
\end{abstract}

The diffusion phenomena of organic solvents in polymers play an important role in many industrial processes, such as polymer processing and drying of solvent coated polymer films. More particularly, estimation of solvent diffusivities is one of the key factors to develop a design methodology for polymeric membrane based organic mixture separation, such as pervaporation (PV) and vapor permeation (VP).

During the past 50 years, the free-volume theory, especially the one developed by Vrentas and Duda, has served as the main basis for the description of transport properties in polymer solutions. ${ }^{1-10}$ The distinct characteristic of this representative model is that there are no adjustable constants and most parameters can be obtained from pure component data. According to this model, the free volume is the controlling factor for solvent jumping in an amorphous rubbery polymer, and the free-volume parameters of some common solvents and polymers have been published, using the viscosity-temperature data. ${ }^{4,10}$ However, due to the lack of viscosity data in the literature, the available free-volume parameters are still insufficient especially for polymers, the physical properties of which are more complicated and uncertain than those of solvents. In recent papers, ${ }^{11,12}$ equations-of-state (EOSs) have been introduced to estimate the free volume of polymer, and the complicated process of measuring polymer viscoelasticity has been avoided. However, it still suffers from the limitation that the characteristic parameters of the polymers summarized in the literature are also limited, which obviously restricts the application of the diffusion model. In addition, predictions of infinite dilution diffusion coefficient, which can provide convincing examination of the reliability of the modified model, are not given. In order to remove these shortcomings, the present work will predict the solvent diffusivities in polymers from group contribution method, using the chemical formula of polymer structural units as input.

\section{EXPERIMENTAL}

According to the free-volume model established by Vrentas and Duda, ${ }^{4,10}$ for an amorphous rubbery polymer-solvent system, the solvent self-diffusion coefficient is given by eq. (1):

$$
\begin{aligned}
D_{1} & =D_{01} \exp \left(-\frac{\omega_{1} \hat{V}_{1}^{*}+\omega_{2} \xi \hat{V}_{2}^{*}}{\omega_{1} \frac{K_{11}}{\gamma}\left(K_{21}+T-T_{\mathrm{g} 1}\right)+\omega_{2} \frac{V_{\mathrm{FH} 2}}{\gamma}}\right) \\
& =D_{01} \exp \left(-\frac{\omega_{1} \hat{V}_{1}^{*}+\omega_{2} \xi \hat{V}_{2}^{*}}{\omega_{1} \frac{K_{11}}{\gamma}\left(K_{21}+T-T_{\mathrm{g} 1}\right)+\omega_{2} \frac{K_{12}}{\gamma}\left(K_{22}+T-T_{\mathrm{g} 2}\right)}\right)
\end{aligned}
$$

${ }^{\dagger}$ To whom correspondence should be addressed (Tel: +86-10-62788777, Fax: +86-10-62770304, E-mail: 1vh103@mails.thu.edu.cn). 
Detailed explanation of the above equation can be found in the literature. ${ }^{1-4}$ Following the previous consideration, ${ }^{11,12}$ the free volume provided by polymer for solvent diffusion, $\hat{V}_{\mathrm{FH} 2} / \gamma$, can be regarded as an alternate portion in the Williams-Landel-Ferry (WLF) equation:

$$
\hat{V}_{\mathrm{FH} 2} / \gamma=V f
$$

where $V$ is the volume of the polymer per gram, and $f$ is the fractional free volume of the polymer.

The Sanchez-Lacombe EOS is chosen to calculate the parameter $V$. In this EOS, holes are introduced in the regular lattice to describe the extra entropy change, and the changes in volume are governed by the changes in the number of holes. In contrast to the SimhaSomcynsky EOS used before, ${ }^{11}$ the Sanchez-Lacombe EOS is not based on a cell model-type partition function, and hence the question of how external and internal degrees of freedom are separated is never encountered. Also, its expression is simple and explicit. The characteristic parameters of the Sanchez-Lacombe EOS can be calculated by the first- and second-order groups in polymer repeating units in the following form: ${ }^{13,14}$

$$
\begin{array}{r}
x=\left(x_{0}+\sum_{i} N_{i} C_{i}+W \sum_{j} M_{j} D_{j}\right) / M_{\mathrm{w}} \\
\left(x=T^{*}, P^{*}, \text { or } \rho^{*}\right)
\end{array}
$$

Here, $x_{0}$ are adjustable parameters which have the same values for all polymers $\left(T_{0}^{*}=1674 \mathrm{~K}, P_{0}^{*}=\right.$ $\left.5225 \mathrm{MPa}, \rho_{0}^{*}=0.405 \mathrm{~g} / \mathrm{cm}^{3}\right), W$ is a constant which is equal to 1 in estimation of the contributions of the second-order groups and equal to 0 when only the first-order groups are contributing, and $M_{\mathrm{w}}$ is the mo- lecular weight of the repeating unit.

In order to determine the fractional free volume, it is assumed that the viscoelastic behavior at atmospheric pressure undergoes an equilibrium isobaric expansion process, as presented elsewhere ${ }^{12,15}$

$$
f=0.025+\int_{T_{\mathrm{g} 2}}^{T} \alpha_{1} \mathrm{~d} T
$$

Here, $\alpha_{1}$ is the thermal expansion coefficient above the glass transition temperature, defined by the following expression:

$$
\alpha_{1}=\frac{1}{V}\left(\frac{\partial V}{\partial T}\right)_{P}=\left(\frac{\partial \ln V}{\partial T}\right)_{P}
$$

By substituting eqs. (4) and (5) into eq. (2), for an amorphous polymer above its glass transition temperature, it follows that

$$
\hat{V}_{\mathrm{FH} 2} / \gamma=V(T)\left[0.025+\ln \frac{V(T)}{V\left(T_{\mathrm{g} 2}\right)}\right]
$$

As stated above, the thermal expansion coefficient reflects the variation of volume with temperature, and thus it plays an important role in the determination of the free volume. In this calculation of $\hat{V}_{\mathrm{FH} 2} / \gamma, \alpha_{1}$ is the dominant factor to account for the increasing free volume with increasing temperature. This parameter is treated as a temperature-dependent parameter, which is in accordance with experimental results. However, the previous treatment of $\hat{V}_{\mathrm{FH} 2} / \gamma$ was based on the utilization of several assumptions, such as assuming $\alpha_{1}$ as a constant. ${ }^{1-4}$ Therefore, it is reasonable to expect this treatment can produce a better agreement than the previous one.

From eqs. (1) and (6), it gives that

$$
D_{1}=D_{01} \exp \left\{-\frac{\omega_{1} \hat{V}_{1}^{*}+\omega_{2} \xi \hat{V}_{2}^{*}}{\omega_{1} \frac{K_{11}}{\gamma}\left(K_{21}+T-T_{\mathrm{g} 1}\right)+\omega_{2} V(T)\left[0.025+\ln \frac{V(T)}{V\left(T_{\mathrm{g} 2}\right)}\right]}\right\}
$$

In this version of the free-volume theory, parameters in the Vrentas-Duda model $\left(D_{01}, \hat{V}_{1}^{*}, \hat{V}_{2}^{*}, K_{11} / \gamma\right.$, $K_{21}-T_{\mathrm{g} 1}, T_{\mathrm{g} 2}$ and $\xi$ ) remain available, while the polymer free-volume parameters $K_{12}$ and $K_{22}$ are no longer required, replaced by using the Sanchez-Lacombe EOS to estimate the polymer hole free volume. The characteristic parameters $\left(T^{*}, P^{*}\right.$ and $\left.\rho^{*}\right)$ of the EOS are the only introduced parameters, and they can be calculated with the group contribution method based on the knowledge of polymer structural units. ${ }^{14} \mathrm{Com}$ pared with the previous work, ${ }^{3,4,10-12}$ this model can predict solvent diffusion coefficients using the poly- mer chemical structures as the input, and the requirement of polymer measurement data can be avoided. It is quite common that the required measurement data in the previous work are not readily available for some polymers, especially for some novel polymers which may not even be synthesized at present. Therefore, the present treatment provides a useful tool from a design point of view, in which the solvent diffusivities can be determined by group contribution method.

In particular, for a polymer-solvent system with vanishing solvent concentration, eq. (7) can be simplified as follows 
Table I. Contributions of first-order groups to the characteristic parameters of the

Sanchez-Lacombe EOS for pure polymers ${ }^{14}$

\begin{tabular}{crcc}
\hline Group & $T_{1 i}^{*}(\mathrm{~K})$ & $P_{1 i}^{*}(\mathrm{MPa})$ & $\rho_{1 i}^{*}\left(\mathrm{~g} \cdot \mathrm{cm}^{-3}\right)$ \\
\hline $\mathrm{CH}_{3}$ & 3364 & 2176 & 6.986 \\
$\mathrm{CH}_{2}$ & 8352 & 3686 & 12.195 \\
$\mathrm{CH}$ & 13983 & 5105 & 18.939 \\
$\mathrm{C}$ & 23329 & 8167 & 27.221 \\
$\mathrm{CH}_{3} \mathrm{COO}$ & 26731 & 29758 & 78.799 \\
$\mathrm{COO}$ & 24634 & 28779 & 71.183 \\
\hline
\end{tabular}

Table II. Contributions of second-order groups to the characteristic parameters of the Sanchez-Lacombe EOS for pure polymers ${ }^{14}$

\begin{tabular}{crcc}
\hline Group & $T_{2 j}^{*}(\mathrm{~K})$ & $P_{2 j}^{*}(\mathrm{MPa})$ & $\rho_{2 j}^{*}\left(\mathrm{~g} \cdot \mathrm{cm}^{-3}\right)$ \\
\hline $\mathrm{C}-\mathrm{CH}_{3} \mathrm{COO}\left(\mathrm{CH}_{2}\right)_{\mathrm{m}}$ & -1354 & 3396 & 1.205 \\
$\mathrm{CH}-\mathrm{COO}\left(\mathrm{CH}_{2}\right)_{\mathrm{m}}$ & -775 & -93 & -2.037 \\
Six-membered ring & 334 & -528 & 0.168 \\
\hline
\end{tabular}

$$
D^{\infty}=D_{01} \exp \left\{-\frac{\xi \hat{V}_{2}^{*}}{V(T)\left[0.025+\ln \frac{V(T)}{V\left(T_{\mathrm{g} 2}\right)}\right]}\right\}
$$

Here, $D^{\infty}$ is the infinite dilution diffusion coefficient. Under the condition of infinite dilution, the free volume contributed by the solvent can be excluded, meaning that the free volume available for solvent jumping is provided all by the polymer. Therefore, this kind of diffusion coefficnet offers a reliable way to evaluate the predictive method for estimating polymer free volume, and it is more convincing than the mutual-diffusion coefficient used before. ${ }^{11,12}$

\section{RESULTS AND DISCUSSION}

The solvent diffusion coefficients for several polymer-solvent systems were calculated and compared with published experimental data, using this model and the Vrentas-Duda model. The parameters of solvent and polymer used in the calculation were given in Tables I-IV.

\section{Prediction of Infinite Dilution Diffusion Coefficient}

Figures 1 and 2 illustrate the calculated results of infinite dilution diffusion coefficients of aromatic solvents in PVAc by both the modified and Vrentas-Duda models. The infinite dilution diffusion coefficients increased with temperature, since increasing temperature facilitated polymer dynamic motions and resulted in more free volume by polymer chain configuration self-adjustment. For PVAc-ethylbenzene, PVAc-toluene and PVAc-o-xylene systems, the predictions by the modified model were more comparable with the
Table III. Solvent free-volume parameters ${ }^{4}$

\begin{tabular}{cccrc}
\hline Solvent & $\begin{array}{c}\hat{V}_{1}^{*} \\
\left(\mathrm{~cm}^{3} \cdot \mathrm{g}^{-1}\right)\end{array}$ & $\begin{array}{c}\left(K_{11} / \gamma\right) \times 10^{3} \\
\left(\mathrm{~cm}^{3} \cdot \mathrm{g}^{-1} \cdot \mathrm{K}^{-1}\right)\end{array}$ & $\begin{array}{c}K_{21}-T_{\mathrm{g} 1} \\
(\mathrm{~K})\end{array}$ & $\begin{array}{c}D_{01} \times 10^{4} \\
\left(\mathrm{~cm}^{2} \cdot \mathrm{s}^{-1}\right)\end{array}$ \\
\hline Benzene & 0.901 & 1.51 & -94.32 & 4.47 \\
Ethylbenzene & 0.928 & 2.22 & -100.81 & 1.54 \\
Heptane & 1.115 & 1.83 & -55.42 & 3.43 \\
Hexane & 1.133 & 1.96 & -41.08 & 3.50 \\
Toluene & 0.917 & 2.20 & -102.72 & 1.87 \\
$o$-Xylene & 1.049 & 1.29 & -53.45 & 4.97 \\
\hline
\end{tabular}

Table IV. Polymer free-volume parameters ${ }^{4,10}$

\begin{tabular}{cccccc}
\hline Polymer & $\begin{array}{c}M_{\mathrm{w}} \\
\left(\mathrm{g} \cdot \mathrm{mol}^{-1}\right)\end{array}$ & $\begin{array}{c}\hat{V}_{2}^{*} \\
\left(\mathrm{~cm}^{3} \cdot \mathrm{g}^{-1}\right)\end{array}$ & $\begin{array}{c}\left(K_{12} / \gamma\right) \times 10^{4} \\
\left(\mathrm{~cm}^{3} \cdot \mathrm{g}^{-1} \cdot \mathrm{K}^{-1}\right)\end{array}$ & $\begin{array}{c}K_{22}-T_{\mathrm{g} 2} \\
(\mathrm{~K})\end{array}$ & $\begin{array}{c}T_{\mathrm{g} 2} \\
(\mathrm{~K})\end{array}$ \\
\hline PBMA & 142.2 & 0.861 & 2.27 & -203.4 & 300 \\
PcHA & 154.2 & 0.805 & 3.65 & -218.0 & 292 \\
PHA & 156.2 & 0.876 & 4.96 & -155.6 & 213 \\
PHMA & 170.3 & 0.889 & 1.68 & -138.6 & 268 \\
PiBA & 128.2 & 0.842 & 5.24 & -196.7 & 230 \\
PVAc & 86.1 & 0.728 & 4.33 & -258.2 & 305 \\
PP & 42.1 & 1.005 & 5.02 & -205.4 & 253 \\
\hline
\end{tabular}

PBMA — poly(butyl methacrylate), PcHA - poly(cyclohexyl acrylate), PHA—poly(hexyl acrylate), PHMA—poly(hexyl methacrylate), PiBA-poly(isobutyl acrylate), PVAc-poly(vinyl acetate), $\mathrm{PP}$-polypropylene.

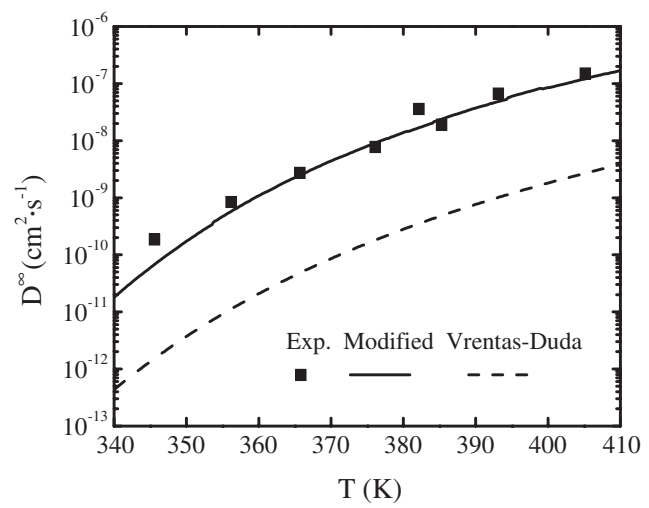

Figure 1. Experimental data ${ }^{16,17}$ and predictions for infinite dilution diffusion coefficients of ethylbenzene in PVAc.

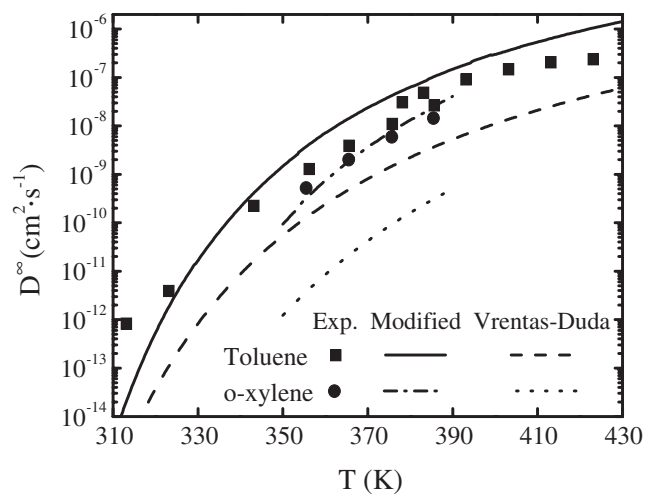

Figure 2. Experimental data ${ }^{16-18}$ and predictions for infinite dilution diffusion coefficients of toluene and $o$-Xylene in PVAc. 


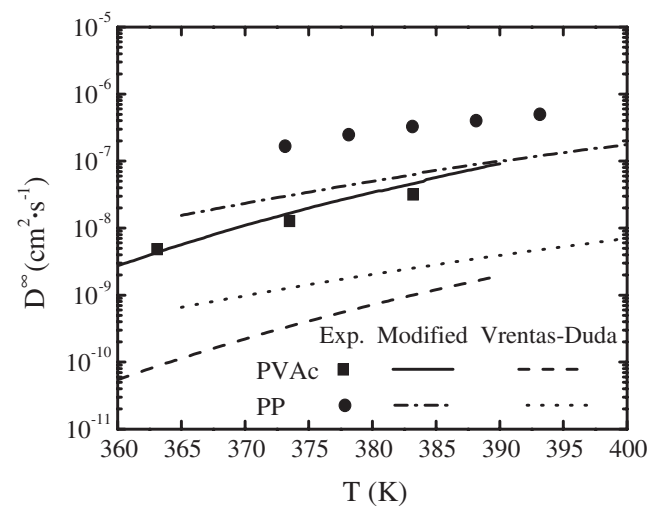

Figure 3. Experimental data ${ }^{17,19}$ and predictions for infinite dilution diffusion coefficients of hexane in PVAc and PP.

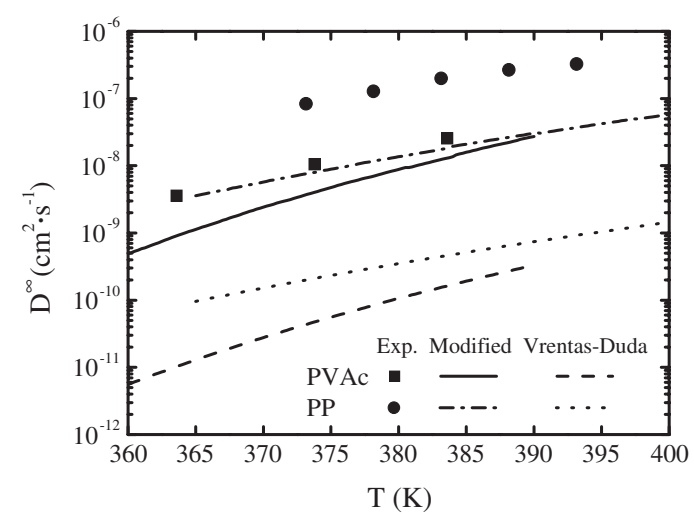

Figure 4. Experimental data ${ }^{17,19}$ and predictions for infinite dilution diffusion coefficients of heptane in PVAc and PP.

experimental data than by the original model. These calculations were done over a broad temperature range, and hence proved suitability of this group contributionbased method. Similar results can be found in PVAcalkane and PP-alkane systems, as shown in Figures 3 and 4. The Vrentas-Duda model produced particular trend as to underestimate the experimental diffusion data in PVAc and PP. In addition, the complex measurements of polymer viscoelasticity must be performed before the calculation. On the contrary, the modified model improved the precision of the estimates without the knowledge of any polymer viscosity data.

It should be pointed out that, the parameter $\xi$ refers to the ratio of the critical molar volume of the solvent jumping unit to that of the polymer jumping unit. This parameter reflects the effect of size and configuration of solvent and polymer, and thus it may need methods from microscopic point of view to give intensive understand, such as molecular modeling. ${ }^{20,21}$ In this study, a widely-used empirical equation was employed to determine $\xi .^{4}$ This empirical equation is more appropriate for relatively symmetrically shaped solvents than for flexible chain solvents. ${ }^{20}$ Therefore, it may be noticed that the diffusion coefficients of alkanes (Figures 3 and 4) exhibit somewhat larger de-

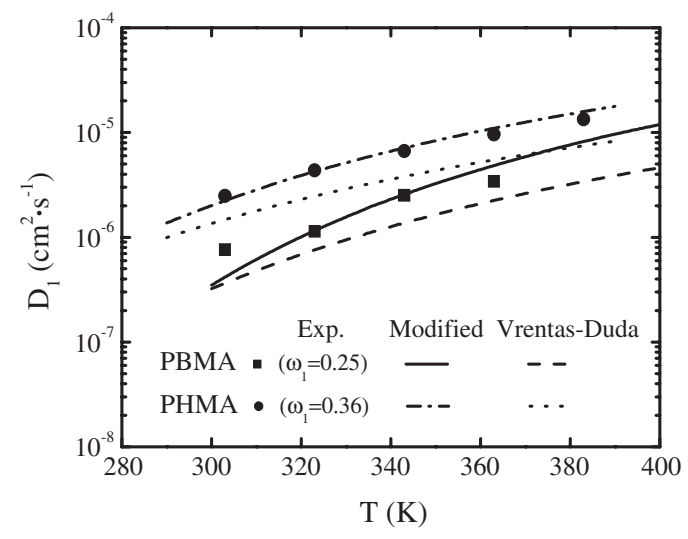

Figure 5. Experimental data ${ }^{10}$ and predictions for benzene self-diffusion coefficients in PBMA and PHMA.

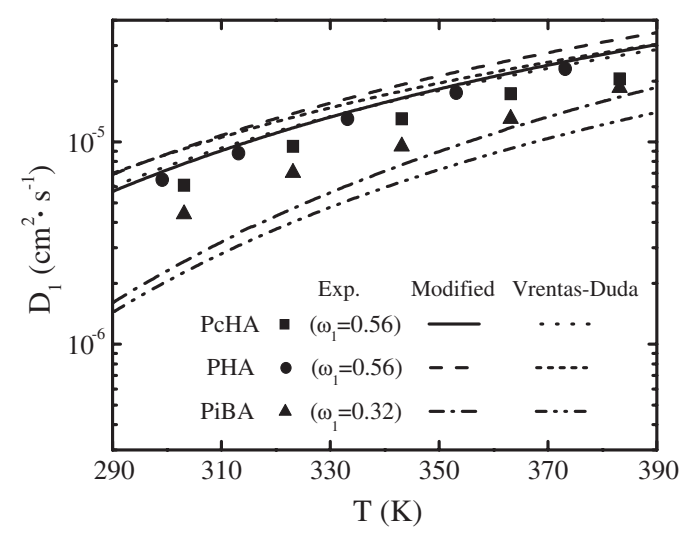

Figure 6. Experimental data ${ }^{10}$ and predictions for benzene self-diffusion coefficients in PcHA, PHA and PiBA.

viations than those of aromatic solvents (Figures 1 and 2). Further research is needed in investigating the effect of size and configuration on diffusion.

\section{Prediction of Solvent Self-Diffusion Coefficient}

The self-diffusion is the molecular migration due to Brownian motions in the absence of concentration gradient. As can be seen in Figure 5, predictions of benzene self-diffusion in two polymethacrylates by the modified model are in a good agreement with experimental results, and more accurate than predictions by the original model. This result can be ascribed to the appropriate estimation of the polymer free volume. As shown in Figure 6, the calculated results for polyacrylates-benzene systems can also reflect the dependence of diffusion coefficients on temperature, although the accuracy is not as satisfied as in most of the systems discussed above. Since PcHA, PHA and PiBA were not very commonly studied polymers, which leaded to the insufficient information for physical properties such as the characteristic parameters, the group contribution method exhibited a promising advantage. Meanwhile, it may be noticed that, for PiBA, the $\mathrm{CH}_{3}(\mathrm{CH}) \mathrm{CH}_{2} \mathrm{CH}_{3}$ group in its repeating 
unit should provide the contribution to the characteristic parameters as second-order group. Unfortunately, the contribution of this group as second-order group was not given in the literature. ${ }^{14}$ Therefore, this study ignored the second-order contribution, and just used the first-order groups in the determination of the characteristic parameters for PiBA. This reason may result in somewhat deviation in the prediction of benzene diffusion coefficients.

\section{CONCLUSIONS}

A modified free-volume model has been proposed by combining the Sanchez-Lacombe EOS with the Vrentas-Duda model to predict the infinite dilution diffusion coefficients and solvent self-diffusion coefficients in amorphous rubbery polymers, without the knowledge of any diffusion or polymer viscosity data. Parameters used in the Vrentas-Duda model remain valid, while the polymer free-volume parameters are no longer used. Only the characteristic parameters of the EOS are introduced and all of them can be estimated by the group contribution method. Compared with the Vrentas-Duda model, the complicated process of measuring polymer viscoelasticity to determine polymer free volume parameters can be avoided. Seven sorts of aromatic and alkane solvents and seven sorts of polymers were used to evaluate the prediction accuracy of the model, and the calculations of diffusion coefficients were generally in a good agreement with the experimental data. Since all the parameters related to polymer can be calculated by group contribution method, this model provides feasibility to correlate molecular diffusion behaviors with polymer chemical structures. It is reasonable to expect this model will be a useful tool in polymer field.

Acknowledgment. We gratefully acknowledge the financial assistance from the Major State Basic Research Development Program of China (973 Program) (No. 2003CB615701) and the National Natural Science Foundation of China (No. 20676068).

\section{LIST OF SYMBOLS}

$D_{01}$ constant pre-exponential factor $\left[\mathrm{cm}^{2} \cdot \mathrm{s}^{-1}\right]$

$D_{1}$ solvent self-diffusion coefficient $\left[\mathrm{cm}^{2} \cdot \mathrm{s}^{-1}\right]$

$D^{\infty}$ infinite dilution diffusion coefficient $\left[\mathrm{cm}^{2} \cdot \mathrm{s}^{-1}\right]$

$f$ polymer fractional free volume

$K_{11}$ solvent free-volume parameter $\left[\mathrm{cm}^{3} \cdot \mathrm{g}^{-1} \cdot \mathrm{K}^{-1}\right]$

$K_{12}$ polymer free-volume parameter $\left[\mathrm{cm}^{3} \cdot \mathrm{g}^{-1} \cdot \mathrm{K}^{-1}\right]$

$K_{21}$ solvent free-volume parameter [K]

$K_{22}$ polymer free-volume parameter [K]

$M_{\mathrm{w}}$ molecular weight of polymer repeating unit $\left[\mathrm{g} \cdot \mathrm{mol}^{-1}\right]$
$T_{\mathrm{g} 1}$ solvent glass transition temperature [K]

$T_{\mathrm{g} 2}$ polymer glass transition temperature $[\mathrm{K}]$

$V$ polymer specific volume $\left[\mathrm{cm}^{3} \cdot \mathrm{g}^{-1}\right]$

$\hat{V}_{1}^{*}$ specific critical hole free volume of solvent required for jump $\left[\mathrm{cm}^{3} \cdot \mathrm{g}^{-1}\right]$

$\hat{V}_{2}^{*}$ specific critical hole free volume of polymer segment required for jump $\left[\mathrm{cm}^{3} \cdot \mathrm{g}^{-1}\right]$

$\hat{V}_{\mathrm{FH} 2}$ polymer specific hole free volume $\left[\mathrm{cm}^{3} \cdot \mathrm{g}^{-1}\right]$

Greek letters

$\alpha_{1}$ thermal expansion coefficient of polymer above glass transition temperature $\left[\mathrm{K}^{-1}\right]$

$\gamma$ overlap factor which accounts for shared free volume

$\xi$ ratio of critical molar volume of solvent jumping unit to that of polymer jumping unit

$\omega_{1}$ solvent weight fraction

$\omega_{2}$ polymer weight fraction

\section{REFERENCES}

1. J. S. Vrentas and J. L. Duda, J. Polym. Sci., Polym. Phys. Ed., 15, 403 (1977).

2. J. S. Vrentas and J. L. Duda, J. Polym. Sci., Polym. Phys. Ed., 15, 417 (1977).

3. J. M. Zielinski and J. L. Duda, AIChE J., 38, 405 (1992).

4. S. U. Hong, Ind. Eng. Chem. Res., 34, 2536 (1995).

5. S. U. Hong, Ind. Eng. Chem. Res., 36, 501 (1997).

6. B. G. Wang, T. Yamaguchi, and S. I. Nakao, J. Polym. Sci., Part B: Polym. Phys., 38, 846 (2000).

7. B. G. Wang, T. Yamaguchi, and S. I. Nakao, J. Polym. Sci., Part B: Polym. Phys., 38, 171 (2000).

8. B. G. Wang, T. Yamaguchi, and S. I. Nakao, Polymer, 42, 5225 (2001).

9. C. L. Zhong, C. S. Yang, and Y. X. Qu, Polym. J., 33, 842 (2001).

10. T. Yamaguchi, B. G. Wang, E. Matsuda, S. Suzuki, and S. I. Nakao, J. Polym. Sci., Part B: Polym. Phys., 41, 1393 (2003).

11. H. L. Lv and B. G. Wang, J. Polym. Sci., Part B: Polym. Phys., 44, 1000 (2006).

12. B. G. Wang, H. L. Lv, and J. C. Yang, Chem. Eng. Sci., 62, 775 (2007).

13. D. Boudouris, L. Constantinou, and C. Panayiotou, Ind. Eng. Chem. Res., 36, 3968 (1997).

14. D. Boudouris, L. Constantinou, and C. Panayiotou, Fluid Phase Equilib., 167, 1 (2000).

15. J. D. Ferry, in "Viscoelastic Properties of Polymers, 3rd ed.," Wiley, New York, 1980.

16. D. S. Hu, C. D. Han, and L. I. Stiel, J. Appl. Polym. Sci., 33, 551 (1987).

17. D. Arnould and R. L. Laurence, Ind. Eng. Chem. Res., 31, 218 (1992).

18. F. Tihminlioglu, R. P. Danner, N. Lutzow, and J. L. Duda, J. Polym. Sci., Part B: Polym. Phys., 38, 2429 (2000).

19. C. W. Zhao, J. D. Li, and C. Y. Zeng, J. Appl. Polym. Sci., 101, 1925 (2006).

20. J. S. Vrentas, C. M. Vrentas, and N. Faridi, Macromolecules, 29, 3272 (1996).

21. H. Ohashi, H. Morito, and T. Yamaguchi, ICOM 2-page Extended Abstracts, 531 (2005). 